\title{
SOME CHARACTERIZATIONS OF INNER PRODUCT SPACES BY INEQUALITIES
}

\author{
Dan Ştefan Marinescu, Mihai Monea and Marian Stroe
}

Abstract. In this paper we present some new characterizations of inner product spaces by using inequalities. First, we explore a classical idea consisting in the transformation of the parallelogram law into an inequality. Then we give some characterizations by using convex and Wright-convex functions.

Mathematics subject classification (2010): 46C15.

Keywords and phrases: Characterization of inner product spaces, norm inequality, convex function, Wright-convex function.

\section{REFERENCES}

[1] J. Alonso, Some remarks on inequalities that characterize inner product spaces, Internat. J. Math. Math. Sci., 15 (1) (1992), 31-34.

[2] C. Alsina, J. Sikorska, M. S. Tomás, Norm Derivatives and Characterizations of Inner Product Spaces, World Scientific Publishing, Singapore, 2010.

[3] H. Angulo, J. Giḿénéz, A. M. Moros, K. Nikodem, On strongly $h$-convex functions, Ann. Funct. Anal., 2 (2011), no. 2, 85-91.

[4] D. AMIR, Characterization of Inner Product Spaces, Birkhäuser Verlag, Basel-Boston, 1986.

[5] Y. Cong-REN, Characterization of inner product spaces, Shuxue Jinzhan (1963), 197-200.

[6] J. G. VAn Der Corput, E. W. Beth, Problem 172, Wisk. Opgaven 16, 421-422 (1937).

[7] F. Dadipour, M. S. Moslehian, A characterization of inner product spaces related to the $p$ angular distance, J. Math. Anal.Appl., 371 (2010), no. 2, 677-681.

[8] M. M. DAY, Some characterizations of inner product spaces, Trans. Amer. Math. Soc., 62 (1947), 320-337.

[9] Z. DARócZy, Z. PÁles, Convexity with given weight sequence, Stocahstica, XI-1 (1987), 5-12.

[10] M. FRÉCHET, Sur la definition axiomatique d'une classe d'espaces vectoriels distanciés applicables vectoriellement sur l'espace de Hilbert, Ann. of Math., 36 (1935), no. 3, 705-718.

[11] N. I. Gurari, Y. I. Sozonov, Normed spaces in which the unit sphere has no bias, Mat. Zametki, 7 (1970), Issue 3, Pag. 307-310.

[12] O. Hirzallah, F. Kittaneh, M. S. Moslehian, Schatten p-norm inequalities related to a characterization of inner product spaces, Math. Ineq. Applic., 13 (2010), no. 2, pp. 235-241.

[13] P. Jordan, J. Von Neumann, On inner products in linear, metric spaces, Ann. of Math., 36 (1935), no. 3, 719-723.

[14] E. R. LORCH, On certain implications which characterize Hilbert space, Ann. of Math., 49 (1948), 523-532.

[15] D. Ş. Marinescu, M. Monea, M. Opincariu, M. Stroe, A characterization of the inner product spaces involving trigonometry, Ann. Funct. Anal., 4 (2013), no. 1, 109-113.

[16] D. Ş. Marinescu, M. Monea, M. Stroe, M. Opincariu, Some equivalent characterizations of inner product spaces and their consequences, Filomat, accepted.

[17] M. S. MosleHIAN, J. M. RASSIAS, A characterization of inner product spaces concerning an EulerLagrange identity, Commun. Math. Anal., 8 (2010), no. 2, 16-21.

[18] M. S. Moslehian, An operator extension of the parallelogram law and related norm inequalities, Math. Ineq. Applic., 14 (2011), no. 3, pp. 717-725. 
[19] M. S. Moslehian, J. M. Rassias, A characterization of inner product spaces, Kochi. J. Math., 6 (2011), 101-101.

[20] K. Nikodem, Z. PÁLes, Characterizations of inner product spaces by strongly convex functions, Banach J. Math. Anal., 5 (2011), no. 1, 83-87.

[21] T. M. Rassias, New characterizations of inner product spaces, Bull Sci. Math., 10 (1984), 95-99.

[22] I. J. SCHOENBERG, A remark on M. M. Day's characterization of inner product spaces and a conjecture of L. M. Blumenthal, Proc. Amer. Math. Soc., 3 (1952), 961-964. 